\title{
Surgical site infection in benign abdominal gynaecological surgeries
}

\author{
Haritha Kizhakke Kodiyath ${ }^{1 *}$, Kunnath Ramakrishnan², Kadaviparambil Jacob ${ }^{3}$
}

\author{
${ }^{1}$ Department of Obstetrics and Gynaecology, Aster Hospital, Mankhool, Dubai \\ ${ }^{2}$ Government Medical College, Ernakulam, Kerala, India \\ ${ }^{3}$ Government Medical College, Manjery, Kerala, India
}

Received: 29 July 2021

Accepted: 01 September 2021

\section{*Correspondence:}

Dr. Haritha Kizhakke Kodiyath,

E-mail: harithapoduval@gmail.com

Copyright: (C) the author(s), publisher and licensee Medip Academy. This is an open-access article distributed under the terms of the Creative Commons Attribution Non-Commercial License, which permits unrestricted non-commercial use, distribution, and reproduction in any medium, provided the original work is properly cited.

\section{ABSTRACT}

Background: Surgical site infection is the commonest complication after gynecological surgeries. Limited studies have been done in India to know the magnitude of the problem. This study aims to know the incidence of surgical site infection, analyze the risk factors and identify the causative organisms.

Methods: A prospective observational study .All women who underwent abdominal surgeries for benign pathologies in department of obstetrics and gynecology, Government medical college, Thrissur from April 2016 to March 2017, were included in the study women more than 80 years old, evidence of infection preoperative or within 48 hours of surgery, laparoscopy procedures unless combined with open surgeries and emergency surgeries were excluded.

Results: The incidence of SSI was found to be $7.9 \%$. Re suturing had to be done in 24 patients. The risk factors found to be significant were age more than 50 years ,patients with education $<7$ th std, diabetes, BMI $>30$,pre hospital stay $>10$ days, ASA score $>3$, duration of surgery $>2$ hours and peri-operative blood transfusion. Staphylococcus aureus was the most common causative organism and was sensitive to amikacin, vancomycin, cloxacillin and its combinations.

Conclusions: SSI pose a significant problem in the post operative period that can affect recovery. It was associated with multiple predisposing factors which could be prevented if these risk factors are identified early and appropriate measures are adopted to support host defense system in the peri operative time.

Keywords: Surgical site infection, Risk factors, Benign gynecological surgeries, Antibiotic sensitivity

\section{INTRODUCTION}

Postoperative surgical site infections (SSI) are an important health care associated infection (HAI) and one of the most frequent causes of post-operative morbidity. SSI is an index of quality health care system. Nosocomial infections and multi drug resistance are one of the major problems in post operative period that prolongs hospital stay which results in loss of time and money.SSI are the third most commonly reported nosocomial infection and they account for a quarter of all nosocomial infections .The overall incidence of wound infection in India varies from $10-33 \%^{1}$. Factors related to host, wound and surgery are the main culprit in the causation of SSI. SSI is a big issue in the developing countries including India compared to other developed countries. This study is relevant in finding the incidence, causative organism and determinants of wound infection helps to reduce SSI by modifying risk factors to change the antibiotic policy.

\section{METHODS}

A prospective observational study conducted in department of obstetrics and gynaecology in Government medical college, Thrissur from April 2016 to March 2017. All women who underwent benign abdominal gynaecological surgeries during the study period were included in the study. Laparoscopic procedures unless 
combined with open surgeries, women with evidence of infection pre operatively and those developing infection within 48 hours of surgeries, emergency/minor procedures and women $>80$ years were excluded. Surgical site was evaluated from third post operative day for any signs of infection till discharge. Patients who developed wound infection after discharge were identified from outpatient clinic, when they came to seek medical advice. Remaining cases were followed up with telephone for signs and symptoms of wound infection. Data was collected by questionnaire as per the proforma and the wounds were graded according to Southampton grading system. A wound is considered to be infected if there were any indurations and swelling discharge, organisms isolated from an aseptically obtained culture, or wound dehiscence within 30 days of operation.

\section{Data analysis}

Data was analyzed using SPSS. Values were expressed in percentage. Association between risk factors and incidence of SSI were assessed by univariate and multivariate logistic regression analysis, $\mathrm{p}<0.05$ was considered significant and $<0.001$ as highly significant.

\section{RESULTS}

Among 453 patients studied the incidence of SSI was $7.9 \% .52 .9 \%$ of the sample were sterile. Staphylococcus was the most common organism followed by E. coli. Routinely we were giving cefotaxime or cefuroxime and metronidazole as prophylaxis. In infected cases and high risk cases cefoperazone+sulbactam and piperacillin+ tazobactam were used. Study showed that staphylococcus was more sensitive to amikacin, vancomycin and cloxacillin and its combinations. E. coli was more sensitive to amikacin and piperacillin. Data showed that, $88 \%$ of women were discharged within the first 7 days and the infection rate was $0.2 \%$. Prolonged stay was due to due to infection. 24 patient had $>15$ days of post operative hospital stay. In this study only 2 cases readmitted for wound infection.

\section{DISCUSSION}

This study is one of the surveillance study on surgical site infection in benign gynecological disease in government medical college, Thrissur, Kerala from April 2016 to March 2017. Sample size was 453, among this 36 patients had wound infection and the incidence was $7.9 \%$.The rate was found to be much less than the studies by Kamat et al and by Pathak et al and high compared to the study conducted by Mahdi et al. ${ }^{1-4}$ The incidence of SSI was found to vary widely depending on the surveillance methods used to identify infections, criteria used to define SSI, and the patient population. All the wound infections were superficial infections which was comparable to the study by Amrita et al. ${ }^{1}$

Table 1: Association between risk factors and wound infection.

\begin{tabular}{|c|c|c|c|c|c|}
\hline Risk factor & Total no. ofpatients & $\mathbf{N}$ & Rate of wound infection (\%) & Chi square & P value \\
\hline \multicolumn{6}{|l|}{ Age (years) } \\
\hline$<50$ & 350 & 25 & 7.1 & \multirow{3}{*}{9.89} & \multirow{3}{*}{0.007} \\
\hline $50-70$ & 95 & 8 & 8.4 & & \\
\hline$>70$ & 8 & 3 & 37.5 & & \\
\hline \multicolumn{6}{|c|}{ Socio economic status } \\
\hline APL & 73 & 2 & 5.55 & \multirow{3}{*}{0.18} & \multirow{3}{*}{0.92} \\
\hline BPL & 378 & 34 & 94.45 & & \\
\hline Tribal & 2 & 0 & 0 & & \\
\hline \multicolumn{6}{|l|}{ Education } \\
\hline$<7$ standard & 127 & 19 & 15 & \multirow{3}{*}{13.76} & \multirow{3}{*}{0.001} \\
\hline $7-12$ & 282 & 17 & 6 & & \\
\hline$>12$ standard & 44 & 0 & 0 & & \\
\hline \multicolumn{6}{|l|}{ Parity } \\
\hline Nulliparous & 53 & 6 & 11.3 & \multirow{2}{*}{0.93} & \multirow{2}{*}{0.33} \\
\hline Multiparous & 400 & 30 & 7.5 & & \\
\hline \multicolumn{6}{|l|}{ BMI } \\
\hline$<25$ & 223 & 6 & 2.7 & \multirow{3}{*}{93.14} & \multirow{3}{*}{$<0.001$} \\
\hline $25-30$ & 200 & 14 & 7 & & \\
\hline$>30$ & 30 & 16 & 51.7 & & \\
\hline \multicolumn{6}{|c|}{ Preoperative hospital stay (days) } \\
\hline$<6$ & 317 & 13 & 4.1 & \multirow{3}{*}{22.51} & \multirow{3}{*}{$<0.001$} \\
\hline $6-10$ & 119 & 19 & 16.0 & & \\
\hline$>10$ & 17 & 4 & 23.5 & & \\
\hline \multicolumn{6}{|c|}{ Previous surgeries } \\
\hline Yes & 98 & 12 & $12.2 \%$ & 3.16 & 0.08 \\
\hline No & 355 & 24 & $6.8 \%$ & & \\
\hline
\end{tabular}


Table 2: Surgical factors associated with infection.

\begin{tabular}{|c|c|c|c|c|c|}
\hline Factors & $\begin{array}{l}\text { Total no. of } \\
\text { patients }\end{array}$ & $\mathbf{N}$ & Rate of wound infection (\%) & Chi square & $P$ value \\
\hline \multicolumn{6}{|l|}{ Anesthesia } \\
\hline GA & 38 & 1 & 2.6 & \multirow{3}{*}{12.74} & \multirow{3}{*}{0.002} \\
\hline Spinal & 335 & 21 & 6.3 & & \\
\hline CSEB & 80 & 14 & 17.5 & & \\
\hline \multicolumn{4}{|l|}{ Surgeon } & \multirow{4}{*}{2.13} & \multirow{4}{*}{0.34} \\
\hline Junior resident & 21 & 2 & 9.5 & & \\
\hline Junior faculty & 23 & 0 & 0 & & \\
\hline Senior faculty & 409 & 34 & 8.3 & & \\
\hline \multicolumn{4}{|l|}{ Operative time (hours) } & \multirow{3}{*}{33.99} & \multirow{3}{*}{$<0.001$} \\
\hline$<2$ & 400 & 21 & 5.3 & & \\
\hline$>2$ & 53 & 15 & 28.3 & & \\
\hline \multicolumn{4}{|l|}{ Blood transfusion } & \multirow{5}{*}{35.02} & \multirow{5}{*}{$<0.001$} \\
\hline Pre operative & 24 & 2 & 8.3 & & \\
\hline Intra operative & 1 & 1 & 100 & & \\
\hline Post operative & 2 & 2 & 100 & & \\
\hline No transfusion & 426 & 31 & 7.3 & & \\
\hline \multicolumn{4}{|l|}{ Closure of incision } & \multirow{4}{*}{0.11} & \multirow{4}{*}{0.95} \\
\hline Continuous transverse & 344 & 28 & 8.1 & & \\
\hline Interrupted transverse & 58 & 4 & 6.9 & & \\
\hline Vertical & 51 & 4 & 7.8 & & \\
\hline \multicolumn{4}{|l|}{ Suture material } & \multirow{4}{*}{2.13} & \multirow{4}{*}{0.34} \\
\hline Ethilon & 19 & 1 & 5.26 & & \\
\hline polygalactin & 421 & 34 & 8.07 & & \\
\hline poliglicaprone & 13 & 1 & 7.69 & & \\
\hline \multicolumn{6}{|l|}{ Co morbidities } \\
\hline Diabetes & 99 & 23 & 23.2 & 40.66 & $<0.001$ \\
\hline Hypertension & 80 & 12 & 15 & 6.61 & 0.01 \\
\hline Anemia corrected & 24 & 2 & 8.3 & 0.37 & 0.55 \\
\hline Thyroid & 50 & 5 & 10 & 0.32 & 0.57 \\
\hline Others & 15 & 6 & 40 & 21.79 & $<0.001$ \\
\hline No co morbidities & 220 & 32 & 14.5 & 25.46 & $<0.001$ \\
\hline \multicolumn{4}{|l|}{ ASA score } & \multirow{4}{*}{62.42} & \multirow{4}{*}{$<0.001$} \\
\hline ASA1 & 252 & 4 & 1.6 & & \\
\hline ASA2 & 186 & 24 & 12.9 & & \\
\hline ASA3 & 15 & 8 & 53.3 & & \\
\hline
\end{tabular}

In this study group, all the wounds were superficial and $80.56 \%$ (29 patients) of the infected wounds were with clear serous discharge. 2 patients $(5.55 \%)$ had induration and erythema, 5 had pus $(13.89 \%)$. Among the infected cases, $32.91 \%$ cured with medical management and $67.08 \%$ underwent for resuturing which was converse to the study by Suwal et al. ${ }^{5}$ In this study $52.9 \%$ of infected cases were sterile.29.4\% of culture contains Staphylococcus, followed by E. coli (8.8\%), MRSA (5.8\%), Klebsiella (2.9\%). Study by Amrita et al found that most of the discharges were sterile and most common organism was E. coli $(36.62 \%)$ followed by mixed infection of $E$. coli and Klebsiella (6.57\%), Pseudomonas (6.57\%), Klebsiella (6.1\%), Staphylococcus (4.69\%). ${ }^{1}$ This study showed Staphylococcus was more sensitive to vancomycin, amikacin and cloxacillin and its combination whereas $E$. coli was more sensitive to amikacin. MRSA was more sensitive to amikacin and vancomycin. Study by Amrita et al amoxyclav was more sensitive in $E$. coli infection.

There are several risk factors associated with wound infection. In our study the wound infection in $<50$ years was $7.1 \%$ and $>70$ years it was raised to $37.5 \%$ and showed age was an important risk factor for wound infection. Result was comparable with the study by Razavi et al the SSI rate of $>65$ years was $33 \%$ whereas $<25$ years the rate was decreased to $4 \%{ }^{6}$ Increased age is associated with co morbidities and poor nutritional status may be the reason for this association. There is a non significant increase in SSI in BPL patient, but $83 \%$ of the study subject belonged to BPL category and the result was comparable with the study by sathyasheela. ${ }^{7}$ 
Table 3: Association between micro organism and antibiotic sensitivity.

\begin{tabular}{|c|c|c|c|c|c|c|c|c|}
\hline Antibiotic & Staphylococcus & $\%$ & E. coli & $\%$ & MRSA & $\%$ & Klebsiella & $\%$ \\
\hline Vancomycin & 4 & 40 & - & & 1 & 50 & - & \\
\hline Amikacin & 5 & 50 & 2 & 66.6 & 1 & 50 & - & \\
\hline Cloxacillin+combi & 6 & 60 & - & & - & & - & \\
\hline Clindamycin & 3 & 30 & - & & - & & 1 & 100 \\
\hline Gentamycin & 2 & 20 & 1 & 33.3 & - & & 1 & 100 \\
\hline Cefaperazone+sulbactam & - & & 1 & 33.3 & - & & - & \\
\hline Doxycycline & 1 & 10 & - & & - & & - & \\
\hline Piperacillin & - & & 2 & 66.6 & - & & - & \\
\hline Ciprofloxacin & 1 & 10 & - & & - & & 1 & 100 \\
\hline Clindamycin & - & & - & & - & & 1 & 100 \\
\hline
\end{tabular}

SSI have an inverse relation with education. In the current study showed that there was a linear relation with increasing BMI . Study by Suwal et al, wound infection in patients with BMI <20 was $13.5 \%$, whereas $\mathrm{BMI}>25$ was $62.2 \%$. Current study showed ASA 1 had $1.6 \%$ of infection whereas in ASA 3 infection increased to $53.3 \%$.Similar result was found in study by Dhamecha et al. ${ }^{8}$ In the present study there was a non significant increase in wound infection among nulliparous women. Conversely Study by Amrita et al, occurrence of postoperative wound infection was more in multiparous women than in nulliparous and they have attributed it to malnutrition and anaemia due to repeated childbirth.

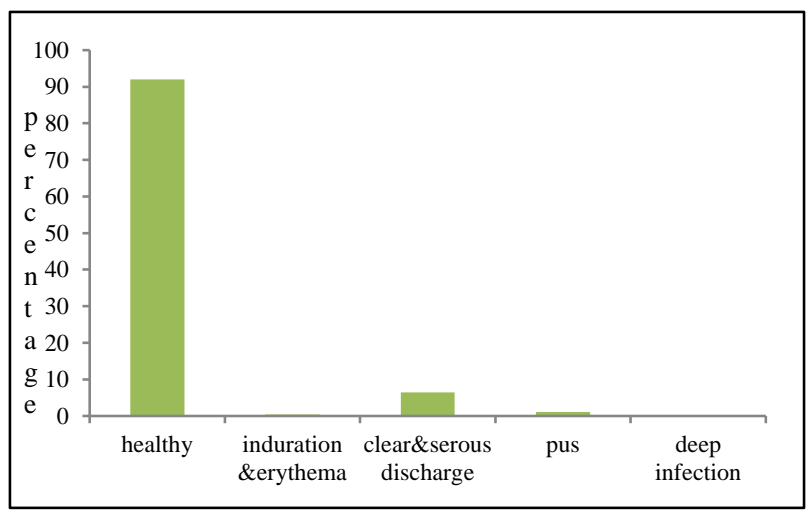

Figure 1: Southampton grading of wound.

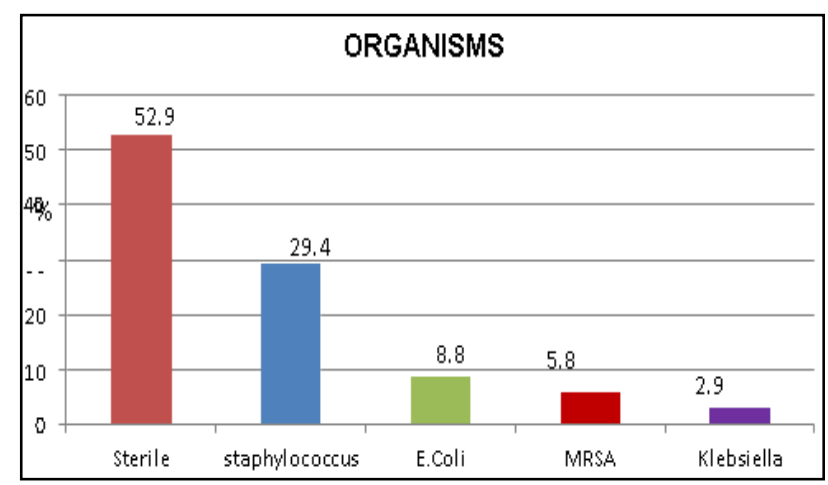

Figure 2: Distribution of micro-organism in pus culture.
In this literature, out of 99 patients with diabetes 23 $(23.3 \%)$ patients got wound infection and it was a significant risk factor of wound infection. Study Amrita et al there is an $8 \%$ risk of wound infection and study by Pathak the rate was $38 \%$ among diabetic patient. Patients with anemia were seemed to be highly susceptible to SSI. Anemia diminishes resistance to infection and is associated with sepsis .In this study $8.3 \%$ of patients with anemia had wound infection, showed minimal non significant increase compared to general population contrary to the study by Nwankwo et al which showed anemia patients have three and half times increased risk for post operative infection compared with patients without anemia and the rate was $30 \% .^{9}$ This study showed a highly significant association between peri operative blood transfusion and wound infection $(18.51 \%$ vs. $7.9 \%$ ). We found that women who had hospital stay of 11 days or more before surgery had high risk of SSI. $23 \%$ of patients with prolonged pre operative stay developed SSI. This may be due to multidrug resistance and increased susceptibility to the hospital acquired infection. Study by Mansour et al showed the wound infection was $18.6 \%$ if the stay was $<15$ days, raised to $25.9 \%$ in patients who had hospital stay $>15$ days. ${ }^{10}$ The rate of infection was high $(17.5 \%)$ with CSEB (combined spinal epidural block). CSEB was used in surgeries which require prolonged operative time. This may be attributed to the increased rate of wound infection among CSEB. General anaesthesia (GA) has least rate of infection $(2.6 \%)$ and spinal anaesthesia (SA) it was $6.3 \%$. Study by Dhamecha et al found that SSI was $16.67 \%$ in GA, which was very high compared to SA where it was only $3.94 \%$. There was a non significant increase in SSI done by the senior faculties compared to junior faculties. This may be because; most of the complicated and high risk cases were done by senior faculty. Study by Dhamecha et al incidence of wound infection was $4.25 \%$. Duration of operation $>2$ hours was associated with increased rate of SSI $(28.3 \%)$ compared to $<2$ hours $(5.3 \%)$. It was not clear whether the risk associated with longer operations is truly due to the length of the operation, or if longer duration is at least in part of the complexity of the operation or operative skill. Study by Seyd et al the SSI rate was $5.4 \%$ when the operation time was $<1.5$ hours and the rate raised to $64 \%$ when the duration of surgery 
was $>4$ hours. ${ }^{6}$ Study by Mahdi et al found operative time $>180$ minutes has a significant effect on wound infection. ${ }^{4}$ In this survey, wound infection in patients with previous history of surgeries was $12.2 \%$ compared to $6.6 \%$ in patients without which is statistically non significant. 402 patients had transverse incision. Out of these 32 got wound infection $(7.9 \%)$ whereas the incidence was $7.8 \%$ in vertical incision which was converse to the study by Vidyadhar et al where the incidence of SSI was highest with vertical midline incision. ${ }^{11}$ In this study 344 cases with transverse incision were closed with continuous sutures, 28 patient had wound infection $(8.1 \%)$ and 4 patients infected in interrupted transverse suture $(6.9 \%)$ among the 58 cases. Study by Suwal et al found that $81.1 \%$ of wound infection with subcuticular suture was $5.4 \%$ with mattress sutures and $13.5 \%$ with staples. ${ }^{5}$ Regarding suture materials, polyglactin was used in 421 surgeries for skin closure $(92.93 \%), 8.07 \%$ got wound infection, poliglecaprone $(7.69 \%)$ and ethilon $(5.26 \%)$ but the difference was not statistically significant. Study by Buresch poliglecaprone 25 has decreased wound infection compared with polyglactin. ${ }^{10}$ Increased wound infection associated with polyglactin may be due to its braiding property. SSI was the most common cause for prolonged hospital stay ( $>15$ days). In this study $83.3 \%$ of subjects with prolonged stay had wound infection. Only 2 cases $(5.55 \%)$ readmitted for wound infection. Study by Aparna et al fifty percent of the patients with infection required repeat hospitalization compared to only $1.98 \%$ patients without infection. Study by Suwal et al the mean post operative wound infection was 11.73 days and the mean post operative hospital stay was $8.57 \%, 38.0 \%$ had 8-14 days of additional hospital stay due to wound infection.

\section{CONCLUSION}

The incidence of SSI was found to be $7.9 \%$. SSI can pose a significant problem in the post operative period and affect the recovery. Multiple factors were found to be predisposing factors for SSI. Increased age more than 50 years, patients with education $<7^{\text {th }}$ std, diabetes, BMI $>30$, pre hospital stay $>10$ days, ASA score $>3$, duration of surgery $>2$ hours and peri operative blood transfusion. Staphylococcus was the most common pathogen isolated. Doctors should strive to identify risk factors which are amenable to correction, to minimize the risk of wound contamination and to support host defence system in the peri-operative time.

\section{ACKNOWLEDGEMENTS}

Authors would like to thank Dr. Sujatha for providing invaluable suggestions and guidance and Mr. Dhatatreya Sangam for statistical analysis.
Funding: No funding sources

Conflict of interest: None declared

Ethical approval: The study was approved by the Institutional Ethics Committee

\section{REFERENCES}

1. Bhadauria AR, Hariharan C. Clinical study of post operative wound infections in obstetrics and gynaecological surgeries in a tertiary care set up. Int $\mathbf{J}$ Reprod Contracept Obstet Gynecol. 2013;2(4):631-8.

2. Kamat AA, Brancazio L, Gibson M. Wound infection in gynecologic surgery. Infect Dis Obstet Gynecol. 2012;8:230-4.

3. Pathak A, Mahadik K, Swam MB. Incidence and risk factors for surgical site infections in obstetric and gynecological surgeries from a teaching hospital in rural India. Antimicrob Resist Infect Control. 2017; 6:66

4. Mahdi H, Goodrich S, Lockhart D. Predictors of surgical site infection in women undergoing hysterectomy for benign gynecologic disease: a multicenter analysis. Natl Surg Quality Improv Data. 2014:21(5):901-9.

5. Suwal A, Shrivastava VR, Giri A. A prospective study of wound infection after laparotomy in obstetrics and gynaecology department. Nepal Med College J. 2012;14(4):267-70.

6. Razavi SM, Ibrahimpoor M, Kashan AS, Jafarian A. Abdominal surgical site infections: incidence and risk factors at an Iranian teaching hospital. Bio Med Central Surg. 2005;5(2):1-5.

7. Sathyaseela MP, Husssain AC, Philip V. Socioeconomic impact on wound management-A pilot study at a Tertiary care Indian Hospital. Wound Med. 2013;2(3):3-5.

8. Dhamecha M, Chauhan N, Kavathi G. Incidence \& predictors of surgical site infections: a study at a tertiary care hospital. GCSMC J Med Sci. 2014:3(2): 25-9.

9. Nwanko EO, Ibeh I, Enabulele OI. Incidence and risk factors of surgical site infection in Kano. Int $\mathrm{J}$ Infect Control. 2012;8:1-6.

10. Buresch AM, Arsdale AV. Comparison of subcuticular suture type for skin closure after cesarean delivery: a randomized controlled trial obstetrics and gynecology. Infect Dis Obstet Gynecol. 2017;130(3): 521-6.

11. Bangal V B, Borawak SK. Study of surgical site infections following gynaecological surgery at tertiary care teaching hospital in rural India. IJBR. 2014;05:27.

Cite this article as: Kodiyath HK, Ramakrishnan K, Jacob K. Surgical site infection in benign abdominal gynaecological surgeries. Int J Reprod Contracept Obstet Gynecol 2021;10:3870-4. 